Bull. Austral. Math. Soc.

Vol. 49 (1994) [35-46]

\title{
ON MORITA DUALITY
}

Weimin XuE

\begin{abstract}
Modules whose nonzero endomorphisms are epimorphisms and modules whose nonzero endomorphisms are monomorphisms are considered in this paper. We prove that these two classes of modules are dual to each other via Morita duality. We also prove that a left artinian ring $R$ with Jacobson radical $J$ has a Morita duality if either (1) $J / J^{2}$ is a central bimodule; or (2) $R$ is artinian right duo and $R / J$ is commutative.
\end{abstract}

Throughout rings are associative with identity and modules are unitary. Module homomorphisms will be written as acting on the side opposite scalars. Tiwary and Pandeya [19] said that a module satisfies the $\left({ }^{*}\right)$ property if each nonzero endomorphism is a monomorphism. Dually, we say that a module has the $\left(^{* *}\right)$ property if each nonzero endomorphism is an epimorphism.

This paper consists of three sections. Modules with property $\left({ }^{* *}\right)$ are considered in the first section. We note that each quotient module of a quasi-projective module with $\left({ }^{* *}\right)$ also has $\left({ }^{* *}\right)$, and the converse is also investigated. Then we discuss the endomorphism rings of modules with property $\left({ }^{* *}\right)$. In Section 2 we prove, under Morita duality, that an reflexive module has the (*)property if and only if its Morita dual has the $\left(^{* *}\right)$ property, and that semihereditary modules and semi-cohereditary modules are also dual to each other. In the last section, we consider artinian rings with a Morita duality. We prove, among other things, that a left artinian ring $R$ with Jacobson radical $J$ has a Morita duality if either (1) $J / J^{2}$ is a central dimodule; or (2) $R$ is artinian right duo and $R / J$ is commutative.

\section{Modules that have PROPERTY **}

The following is the dual result of $[19$, Theorem 1.5].

THEOREM 1. Let $R$ be a ring.

(1) If ${ }_{R} P$ is a quasi-projective module with the property $\left({ }^{* *}\right)$, then each quotient module of ${ }_{R} P$ has $\left({ }^{* *}\right)$.

(2) Suppose that ${ }_{R} P \stackrel{P}{\longrightarrow}{ }_{R} M \longrightarrow 0$ is a projective cover such that $\operatorname{Ker}(p)$ is stable under the endomorphisms of $P$ and $\operatorname{Hom}_{R}(P, \operatorname{Ker}(p))=0$. If ${ }_{R} M$ has the property $\left({ }^{* *}\right)$, then so does ${ }_{R} P$.

Received 21 January 1993

Copyright Clearance Centre, Inc. Serial-fee code: 0004-9729/94 \$A2.00+0.00. 
Proof: (1) Let ${ }_{R} M$ be a quotient module of ${ }_{R} P$ and $p:{ }_{R} P \longrightarrow{ }_{R} M$ be the natural epimorphism. If $M$ does not have $\left({ }^{* *}\right)$, there exists $0 \neq f \in \operatorname{End}\left({ }_{R} M\right)$ such that $f$ is not an epimorphism. Since ${ }_{R} P$ is quasi-projective, there is a $g \in \operatorname{End}\left({ }_{R} P\right)$ with the diagram

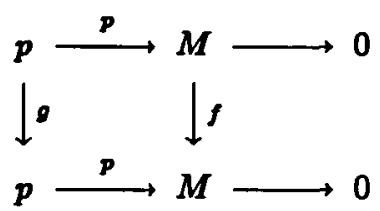

commutative. It follows that $g \neq 0$ and $g$ is not an epimorphism. This contradicts that ${ }_{R} P$ has property $\left({ }^{* *}\right)$.

(2) Assume that there exists a $0 \neq g \in \operatorname{End}\left({ }_{R} P\right)$ and $g$ is not an epimorphism. Since $\operatorname{Ker}(p)$ is stable under the endomorphisms of ${ }_{R} P, h=\left.g\right|_{\operatorname{Ker}(p)} \in \operatorname{End}(\operatorname{Ker}(p))$. It follows that there is an $f \in \operatorname{End}\left({ }_{R} M\right)$ such that the diagram

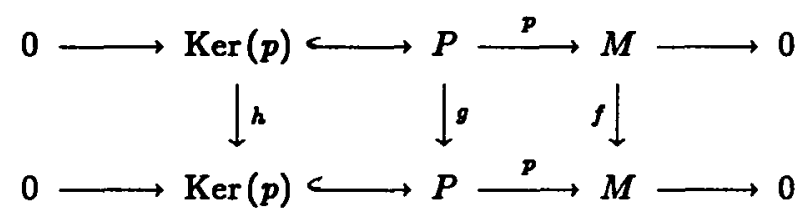

is commutative (see [16, p.27], for example). Now $g \neq 0$ and $\operatorname{Hom}_{R}(P, \operatorname{Ker}(p))=0$, hence $\operatorname{Im}(g)$ is not contained in $\operatorname{Ker}(p)$. Therefore $f \neq 0$. Since $(P, p)$ is a projective cover of $M$ and $g$ is not an epimorphism, $g p$ is not onto. So $p f$ is not onto, neither is $f$. This is a contradiction since ${ }_{R} M$ has $\left({ }^{* *}\right)$.

It is easy to see that every module with either property $\left({ }^{*}\right)$ or $\left({ }^{* *}\right)$ must be indecomposable.

\section{PROPOSITION 2.}

(1) If ${ }_{R} P$ is a quasi-projective module with $\left({ }^{* *}\right)$, then $\operatorname{End}\left({ }_{R} P\right)$ is a division ring.

(2) If ${ }_{R} Q$ is an quasi-injective module with (*), then $\operatorname{End}\left({ }_{R} Q\right)$ is a division ring.

Proof: (1) Let $0 \neq f \in \operatorname{End}\left({ }_{R} P\right)$ be an epimorphism. Since ${ }_{R} P$ is quasiprojective, the exact sequence

$$
0 \longrightarrow \operatorname{Ker}(f) \hookrightarrow P \stackrel{f}{\longrightarrow} P \longrightarrow 0
$$

splits. Having property (**), ${ }_{R} P$ is indecomposable, so $\operatorname{Ker}(f)=0$ and $f$ is an isomorphism. 
(2) Let $0 \neq g \in$ End $\left({ }_{R} Q\right)$ be a monomorphism. Then $0 \neq \operatorname{Im}(g) \cong_{R} Q$, which is indecomposable since ${ }_{R} Q$ has $\left(^{*}\right)$. Since ${ }_{R} Q$ is quasi-injective, $\operatorname{Im}(g)$ is a summand of ${ }_{R} Q$ and then $\operatorname{Im}(g)=Q$. That is, $g$ is an isomorphism.

The following proposition is the analogous result of [19, Proposition 2.8].

Proposition 3. Suppose that ${ }_{R} P \stackrel{p}{\longrightarrow} M \longrightarrow 0$ is a projective cover of a simple module ${ }_{R} M$. If $\operatorname{Hom}_{R}(P, J P)=0$, then $\operatorname{End}\left({ }_{R} P\right)$ is a division ring, where $J=J(R)$ is the Jacobson radical of $R$.

Proof: Since $\operatorname{Ker}(p)=J P$ is the unique maximal submodule of ${ }_{R} P$ and $\operatorname{Hom}_{R}(P, J P)=0,{ }_{R} P$ has $\left(^{* *}\right)$ by Theorem 1. Now the result follows from Proposition 2.

Following Shrikhande [18], we call a Module ${ }_{R} P$ (semi-) hereditary in case each (finitely generated) submodule of ${ }_{R} P$ is projective, and call a module ${ }_{R} Q$ (semi) cohereditary in case each (finitely cogenerated) factor module of ${ }_{R} Q$ is injective.

\section{Proposition 4.}

(1) An indecomposable hereditary module ${ }_{R} P$ has property (*); and

(2) an indecomposable cohereditary module ${ }_{R} Q$ has property $\left(^{* *}\right)$.

Proof: (1) If $0 \neq f \in \operatorname{End}\left({ }_{R} P\right)$, then $\operatorname{Ker}(f)(\neq P)$ is a summand of ${ }_{R} P$ since $\operatorname{Im}(f)$ is projective. Hence $\operatorname{Ker}(f)=0$. (2) If $0 \neq g \in \operatorname{End}\left({ }_{R} Q\right)$, then $0 \neq \operatorname{Im}(g) \cong$ $Q / \operatorname{Ker}(g)$ is injective. So $\operatorname{Im}(g)$ is a summand of ${ }_{R} Q$ and hence $\operatorname{Im}(g)=Q$.

Corollary 5. Let ${ }_{R} M$ be an indecomposable module. If ${ }_{R} M$ is both hereditary and cohereditary, then $\operatorname{End}\left({ }_{R} M\right)$ is a division ring.

If $R$ is a left perfect ring and ${ }_{R} P$ is an indecomposable hereditary module, then End $\left({ }_{R} P\right)$ is a division ring [9, Proposition 1.2]. Now we show the following.

Corollary 6 . Let ${ }_{R} Q$ be an indecomposable cohereditary module. If ${ }_{R} Q$ is noetherian, then $\operatorname{End}\left({ }_{R} Q\right)$ is a division ring.

Proof: By Proposition 4, ${ }_{R} Q$ has the property $\left({ }^{* *}\right)$. Hence each nonzero endomorphism of ${ }_{R} Q$, being an epimorphism, is an isomorphism by [1, Lemma 11.6(2)].

\section{On Morita duality}

Morita duality was established by Morita [13] and Azumaya [2]. A presentation of this duality can be found in Anderson and Fuller [1, Sections 23 and 24], or the author's recent book [25]. Recall that a bimodule ${ }_{R} E_{S}$ defines a Morita duality if ${ }_{R} E_{S}$ is a faithfully balanced bimodule such that both ${ }_{R} E$ and $E_{S}$ are injective cogenerators; and in this case the natural domain and range of such a duality are the categories of $E$-reflexive modules. 
Theorem 7. Let ${ }_{R} E_{S}$ define a Morita duality and ${ }_{R} P$ be an $E$-reflexive module. Then ${ }_{R} P$ has property $\left({ }^{*}\right)$ if and only if $P_{S}^{*}=\operatorname{Hom}_{R}\left({ }_{R} P,{ }_{R} E_{S}\right)$ has property $\left(^{* *}\right)$.

Proof: Since ${ }_{R} P$ is $E$-reflexive, the evaluation map ${ }_{R} P \longrightarrow{ }_{R} P^{* *}=$ $\operatorname{Hom}_{S}\left(P_{S}^{*},{ }_{R} E_{S}\right)$ is an isomorphism.

( ). Let $0 \neq f \in \operatorname{End}\left({ }_{R} P\right)$. Since ${ }_{R} E$ is an injective cogenerator, we have $0 \neq f^{*} \in \operatorname{End}\left(P_{S}^{*}\right)$, where $f^{*}: g \mapsto f g$ for $g \in P_{S}^{*}$. So $f^{*}$ is an epimorphism. If there exists $0 \neq x \in \operatorname{Ker}(f)$, it follows from the injective cogenerator of ${ }_{R} E$ that there is $0 \neq g \in P_{S}^{*}$ such that $(x) g \neq 0$. Now $f^{*}$ is epic, so $g=f h$ for some $h \in P_{S}^{*}$. Then $0 \neq(x) g=(x) f h=0$, since $x \in \operatorname{Ker}(f)$. This contradiction shows that $\operatorname{Ker}(f)=0$ and $f$ is a monomorphism.

$(\Longrightarrow)$ Let $0 \neq f \in \operatorname{End}\left(P_{S}^{*}\right)$. Then $0 \neq f^{*} \in \operatorname{End}\left({ }_{R} P^{* *}\right)$, since $E_{S}$ is an injective cogenerator. Being isomorphic to ${ }_{R} P,{ }_{R} P^{* *}$ has property $\left({ }^{* *}\right)$ and so $f^{*}$ is a monomorphism. It follows that $\operatorname{Hom}_{S}\left(P^{*} / \operatorname{Im}(f), E_{S}\right)=0$. Now $E_{S}$ is a cogenerator, hence $P^{*} / \operatorname{Im}(f)=0$ and $P^{*}=\operatorname{Im}(f)$.

The notion of linearly compact modules (artinian modules are linearly compact) plays an important role in the duality theory [15]. The basic properties of linearly compactness are given in [25, Section 3]. Mueller [15] proved that if ${ }_{R} E_{S}$ defines a Morita duality then the linearly compact left $R$ - (or right $S$-) modules are precisely the $E$-reflexive modules.

The following result is a generalisation of Fisher $[8$, p.391, Lemma] which asserts that an artinian projective module is fininitely generated. Fisher [8, p.391, Example] gave an artinian projective module which is not noetherian.

Lemma 8. If ${ }_{R} P$ is a linearly compact projective module, then ${ }_{R} P$ is finitely generated.

Proof: Let $J=J(R)$. By [25, Proposition 3.11], ${ }_{R} P$ is complemented in the sense that for any submodule $N$ of $P$ there is a submodule $L$ of $P$ such that $L$ is minimal with respect to the property that $N+L=P$. It follows from [11, Theorems 11.1.5 and 11.1.7] that $J P$ is small in $P$. Now $P / J P$ is linearly compact and semisimple, so it must be finitely generated. Hence ${ }_{R} P$ is finitely generated.

It follows immediately from Lemma 8 that a linearly compact hereditary module is noetherian. In particular, a left hereditary ring with a duality must be left noetherian.

Theorem 9. Let ${ }_{R} E_{S}$ define a Morita duality and ${ }_{R} P$ be an $E$-reflexive module.

(1) If ${ }_{R} P$ is (semi-) hereditary, then $P_{S}^{*}=\operatorname{Hom}_{R}\left({ }_{R} P,{ }_{R} E_{S}\right)$ is (semi-) cohereditary;

(2) If ${ }_{R} P$ is semi-cohereditary, then $P_{S}^{*}=\operatorname{Hom}_{R}\left({ }_{R} P,{ }_{R} E_{S}\right)$ is semihereditary. 
Proof: (1) If $M_{S}$ is a (finitely cogenerated) factor module of $P_{S}^{*}$, then ${ }_{R} M^{*}=$ $\operatorname{Hom}_{S}(M, E)$ (is finitely generated and) can be embedded into ${ }_{R} P^{* *}=\operatorname{Hom}_{S}\left(P^{*}, E\right)$ $\cong{ }_{R} P$. Hence ${ }_{R} M^{*}$ is projective. Being a factor module of the $E$-reflexive module $P_{S}^{*}, M_{S}$ is $E$-reflexive. Then $R_{R} M^{*}$ is $E$-reflexive, so it is linearly compact. By the above lemma, $R^{M^{*}}$ is finitely generated. Hence $M_{S} \cong \operatorname{Hom}_{R}\left(M^{*}, E\right)_{S}$ is an injective $S$-module by [1, Theorem 24.6]. This shows that $P_{S}^{*}$ is a (semi-) cohereditary module.

(2) Let $M_{S}$ be a finitely generated submodule of $P_{S}^{*}$. Then ${ }_{R} M^{*}$ is a finitely cogenerated factor module of ${ }_{R} P^{* *} \cong{ }_{R} P$, so ${ }_{R} M^{*}$ is injective. It follows from [1, Theorem 24.6] that $M_{S} \cong \operatorname{Hom}_{R}\left(M^{*}, E\right)$ is a projective $S$-module. Therefore $P_{S}^{*}$ is semihereditary.

The following question arises naturally from Theorem 9: Let ${ }_{R} E_{S}$ define a Morita duality and ${ }_{R} Q$ be an $E$-reflexive module. If ${ }_{R} Q$ is cohereditary, is $Q_{S}^{*}=$ $\operatorname{Hom}_{R}\left({ }_{R} Q,{ }_{R} E_{S}\right)$ hereditary? We answer it in the negative as follows.

EXAMPLE 10. Let $R=F[[x]]$ be the formal power series ring over a field $F$. As abelian group, let $E=F\left[x^{-1}\right]$, the polynomial ring over $F$ with indeterminate $x^{-1}$. $E$ obtains an $R$-module structure by additive extension of

$$
\left(a_{j} x^{j}\right)\left(a_{i} x^{-i}\right)= \begin{cases}0 & \text { if } i<j \\ a_{j} a_{i} x-i+j & \text { otherwise }\end{cases}
$$

We note that the artinian $R$-module ${ }_{R} E_{R}$ defines a Morita duality. Let $Q=F((x))$ be the quotient field of $R$. Then the $R$-module $Q$ is $E$-reflexive and cohereditary. Since ${ }_{R} Q$ is not finitely cogenerated, its dual $Q_{R}^{*}=\operatorname{Hom}_{R}(Q, E)_{R}$ is not finitely generated. Hence $Q_{R}^{*}$ is not projective by Lemma 8 .

\section{Artinian Rings with Morita duality}

Azumaya [2] and Morita [13] proved that a left artinian ring $R$ possesses a (left) duality if and only if $R$ has an artinian injective cogenerator ${ }_{R} E$. In this event, ${ }_{R} E$ induces a duality between finitely generated left $R$-modules and finitely generated right End $\left({ }_{R} E\right)$-modules. They also verified the existence of a duality for commutative artinian rings. In this section, we always denote the radical of a ring $R$ by $J$. If ${ }_{R} M$ is a left $R$-module, we let $r_{M}(J)=\{m \in M \mid J m=0\}$.

Rosenberg and Zelinsky [17] have proved that a left artinian ring $R$ has a duality if and only if $R / J^{2}$ does. Using this result one concludes that $R$ has a duality if $R / J^{2}$ is commutative. In this paper, we first generalise this result by showing that $R$ still has a duality if $J / J^{2}$ is only a central $R$-bimodule. Employing a result of Hill [10] we prove that an artinian right duo ring $R$ has a duality in case $R / J$ is commutative. 
It is well-known that a left perfect ring with a duality is left artinian. We generalise this to rings whose linearly compact left modules have projective covers. In the conclusion, we partially solve a problem raised in our paper [24].

We begin with a lemma which may be known. Here we give its proof, since we are unable to find any proof in the literature. Recall that a module is artinian if and only if each factor module is finitely cogenerated.

LEMma 11. If $E$ is a finitely cogenerated module but not artinian, then there is a non-zero artinian submodule $E_{0}$ of $E$ such that $E / E_{0}$ is not finitely cogenerated but $E / E_{1}$ is finitely cogenerated for each proper submodule $E_{1}$ of $E_{0}$.

Proof: Let

$$
W=\left\{E^{\prime} \leqslant E \mid E / E^{\prime} \text { is not finitely cogenerated }\right\}
$$

Then $0 \notin W$, since $E$ is finitely cogenerated. And $W$ is non-empty, since $E$ is not artinian. Partially order $W$ by the opposite of inclusion. Consider a totally ordered subset $\left\{E_{i}\right\}_{i \in I}$ of $W$. Clearly we have $\bigcap_{j \in I}\left(E_{j} / \bigcap_{i \in I} E_{i}\right)=0$. If $E / \bigcap_{i \in I} E_{i}$ is finitely cogenerated, then there is a finite subset $\left\{j_{1}, \ldots, j_{n}\right\}$ of $I$ such that $\bigcap_{k=1}^{n}\left(E_{j_{k}} / \bigcap_{i \in I} E_{i}\right)=$ 0 . Since $\left\{E_{i}\right\}_{i \in I}$ is totally ordered, $\bigcap_{k=1}^{n} E_{j_{k}}=E_{j_{t}}$ for some $t, 1 \leqslant t \leqslant n$. It follows that $0=\bigcap_{k=1}^{n}\left(E_{j_{k}} / \bigcap_{i \in I} E_{i}\right)=E_{j_{t}} / \bigcap_{i \in I} E_{i}$ and $E_{j_{t}}=\bigcap_{i \in I} E_{i}$. This is a contradiction, since $E_{j_{t}} \in W$. Hence $E / \bigcap_{i \in I} E_{i}$ is not finitely cogenerated, and then $\bigcap_{i \in I} E_{i} \in W . \mathrm{By}$ Zorn's lemma, $W$ has a maximal element $E_{0}$, which is actually minimal with respect to inclusion. If $E_{1}$ is a proper submodule of $E_{0}$, then by the minimality of $E_{0}$ we have $E_{1} \notin W$. Hence $E / E_{1}$ is finitely cogenerated, and so is $E_{0} / E_{1}$. It follows that $E_{0}$ is artinian.

A bimodule ${ }_{R} M_{R}$ is called central if ${ }_{R} M$ (or $M_{R}$ ) has a generating set $\left\{m_{i}\right\}_{i \in I}$ such that $r m_{i}=m_{i} r$ for each $r \in R$ and $i \in I$, and $\left\{m_{i}\right\}_{i \in I}$ is called a central generating set for ${ }_{R} M_{R}$. For example, any module ${ }_{R} M$ over a commutative ring $R$ becomes a central bimodule ${ }_{R} M_{R}$ if we define $M_{R}$ via $m \cdot r=r m$ in ${ }_{R} M$.

Theorem 12. A left artinian ring $R$ has a duality if $J / J^{2}$ is a central $R$ bimodule.

Proof: We may assume $J^{2}=0$, since $R$ is left artinian. Also we have a finitely cogenerated injective cogenerator ${ }_{R} E$. We shall prove that ${ }_{R} E$ is artinian.

If not, by Lemma 11, $E$ has a non-zero artinian submodule $E_{0}$ with the property stated in the lemma. Since $R$ is left noetherian and ${ }_{R} J_{R}$ is a central bimodule, ${ }_{R} J_{R}$ 
has a finite central generating set $\left\{j_{1}, \ldots, j_{n}\right\}$. Define a mapping

via

$$
\begin{aligned}
f: \operatorname{Soc}\left(E / E_{0}\right)=r_{E / E_{0}}(J) & \rightarrow\left(E_{0} / J E_{0}\right)^{n} \\
e+E_{0} & \mapsto\left(j_{i} e+J E_{0}\right)_{i=1}^{n} .
\end{aligned}
$$

Then $f$ is an $R$-homomorphism. Since $E_{0} \neq 0$ and $R$ is left artinian, $J E_{0}$ is a proper submodule of $E_{0}$. So $E / J E_{0}$ is finitely cogenerated by Lemma 11 , and then $r_{E / J E_{0}}(J)=\operatorname{Soc}\left(E / J E_{0}\right)$ is finitely generated. Now $\operatorname{Ker}(f)=\left\{e+E_{0} \in E / E_{0} \mid J e \subseteq\right.$ $\left.J E_{0}\right\}$, which is finitely generated, since there is an epimorphism from $r_{E / J E_{0}}(J)$ to $\operatorname{Ker}(f)$ given by $e+J E_{0} \mapsto e+E_{0}$. And $\operatorname{Im}(f)$, being a submodule of the finitely generated semisimple module $\left(E_{0} / J E_{0}\right)^{n}$, is finitely generated. Therefore $\operatorname{Soc}\left(E / E_{0}\right)$ is finitely generated, and then $E / E_{0}$ is finitely cogenerated, since $R$ is left artinian. This contradicts to the property of $E_{0}$.

COROLlary 13. A left artinian ring $R$ has a duality if $R / J^{2}$ is commutative; in particular, a commutative artinian ring has a duality.

To see that our Theorem 12 is a non-trivial generalisation, we construct an artinian ring $R$ with $J^{2}=0$ such that ${ }_{R} J_{R}$ is a central bimodule but $R$ is not commutative. Let $D$ be a non-commutative division ring and $n$ a positive integer. Let

$$
R=D \times D^{n}
$$

as abelian group and define the multiplication via

$$
\left(d_{1}, m_{1}\right)\left(d_{2}, m_{2}\right)=\left(d_{1} d_{2}, d_{1} m_{2}+m_{1} d_{2}\right) .
$$

Then $R$ is the ring we want. One notes that $R$ is an exact ring in the sense of Azumaya [3].

In [17, p.375], Rosenberg and Zelinsky gave an example of a local left artinian (not right artinian) ring $R$ such that $R / J$ is a field, but $R$ does not possess a duality. Using Cohn's division ring extensions [5], one constructs an artinian ring without a duality (see $[1$, p.286]). This artinian ring is not commutative modulo its radical. From these two examples the following question arises naturally.

Open question: If $R$ is an artinian ring and $R / J$ is commutative, does $R$ have a duality?

A ring is called left (right) duo if each left (right) ideal is a two-sided ideal. The above question has an affirmative answer if $R$ is a left duo ring [22]. Next we show that the answer is still "Yes" if $R$ is a right duo ring.

Theorem 14. If $R$ is an artinian right duo ring with $R / J$ commutative, then $R$ has a duality. 
Proof: By Courter [6, Theorem 3.1] we may assume that $R$ is a local ring, and by [17] we suppose $J^{2}=0$. We need to show that the composition length of ${ }_{R} E=E\left({ }_{R}(R / J)\right), c\left({ }_{R} E\right)$, is finite.

(1) If $c\left(J_{R}\right)=1$, by Hill [10, Theorem 3.2] we have $c(R E)=2$.

(2) Now let $c\left(J_{R}\right)=n>1$ and write $J_{R}=\bigoplus_{i=1}^{n} j_{i} R$. Since $R$ is right duo, each $j_{i} R$ is an ideal. By $[1$, p.286] we have

$$
{ }_{R} E / \operatorname{Soc}\left({ }_{R} E\right) \cong{ }_{R} \operatorname{Hom}_{R}\left({ }_{R} J,{ }_{R}(R / J)\right) \cong \bigoplus_{i=1}^{n}{ }_{R} \operatorname{Hom}_{R}\left({ }_{R}\left(j_{i} R\right),{ }_{R}(R / J)\right)
$$

which has length $n$ since we can apply the result of (1) to the ring $\boldsymbol{R}_{i}=$ $R /\left(\bigoplus_{k \neq i} j_{k} R\right)$ since $c\left(J\left(R_{i}\right)_{R_{i}}\right)=1$, where $i=1, \ldots, n$. It follows that $c\left({ }_{R} E\right)=n+1$ is finite.

Corollary 15. If $R$ is a local artinian right duo ring with $c\left(\left(J / J^{2}\right)_{R}\right)>1$, then $R$ has a duality.

Proof: By [22, Theorem 2(1)], $R / J$ is a field.

As a generalisation of left perfect ring, we call a ring $R$ left weakly perfect in case each linearly compact left $R$-module has a projective cover. We have

Propositron 16. The following are equivalent for a semiperfect ring $R$ :

(1) $R$ is left weakly perfect;

(2) Every non-zero linearly compact left $R$-module has a maximum submodule;

(3) Every linearly compact left $R$-module is finitely generated;

(4) Every linearly compact left $R$-module is noetherian.

Proof: (1) $\Rightarrow(2)$. [4, Proposition 2].

(2) $\Rightarrow(3)$. If ${ }_{R} M \neq 0$ is linearly compact, then $M / J M$ is linearly compact and finitely generated. Let $M=K+J M$ where $K$ is finitely generated. Then $J(M / K)=M / K$ which is still linearly compact. Hence $M / K=0$ and $M=K$.

(3) $\Rightarrow(4)$. Submodules of a linearly compact module are linearly compact.

$(4) \Rightarrow(1)$. A ring is semiperfect if and only if each noetherian module has a projective cover.

It is known that a left perfect ring with a duality is left artinian. A slight generalisation is given as follows. 
THEorem 17. If $R$ is a left weakly perfect ring with a duality, then $R$ is left artinian.

Proof: Let ${ }_{R} E_{S}$ define a Morita duality. Since ${ }_{R} E$ is linearly compact, it is noetherian by Proposition 16. So $S=\operatorname{End}\left({ }_{R} E\right)$ is a semiprimary ring by [7, Corollary 2.5]. Then $S$ is right artinian and $R$ is left artinian.

A ring extension $R \leqslant S$ is called a finite triangular extension if there are a finite number of elements $s_{1}, \ldots, s_{n}$ of $S$ such that $S=\sum_{i=1}^{n} R s_{i}$ and $\sum_{i=1}^{j} R s_{i}=\sum_{i=1}^{j} s_{i} R$ $(j=1, \ldots, n)$. Finite triangular extensions arise from Azumaya's exact rings [3] which are such over a finite direct sum of local exact rings [23, Theorem 3]. Lemonnier [12, Theorem 5.4] showed that a finite triangular extension over a ring with a duality has itself a duality. But the converse is open (see [24, p.59]) and is far from being solved. We shall give partial answers.

Lemma 18. Let $R \leqslant S$ be a ring extension and ${ }_{R} E$ a left injective $R$-module. If ${ }_{R} \operatorname{Hom}_{R}(S, E)$ is artinian (noetherian), then so is ${ }_{R} E$.

Proof: Since ${ }_{R} E$ is injective, the embedding of $R$-modules

$$
R \hookrightarrow S
$$

induces an epimorphism of left $R$-modules

$$
{ }_{R} \operatorname{Hom}_{R}(S, E) \longrightarrow \operatorname{Hom}_{R}(R, E) \cong{ }_{R} E .
$$

Recall that a bimodule ${ }_{R} E_{S}$ defines a duality if and only if $R$ is a left linearly compact ring, $R E$ is a linearly compact and finitely cogenerated injective cogenerator, and $S \cong \operatorname{End}\left({ }_{R} E\right)$ canonically, (see $[15,20$, or 25]). In this event we also say that $R$ has a duality induced by ${ }_{R} E$ or that ${ }_{R} E$ defines a duality.

Proposition 19. Let $R \leqslant S$ be a finite triangular extension and $S$ have a duality. If $\bigcap_{n=1}^{\infty} J(S)^{n}=0$ and $R$ is left linearly compact, then $R$ is a left noetherian ring with a duality.

Proof: Let ${ }_{R} U$ define a duality. By Mueller [14, Theorem 7], $s U$ is artinian and $S$ is left noetherian. Hence $R$ is left noetherian by [21, Theorem 5.2]. Since a left linearly compact ring must be semiperfect, we have a finitely cogenerated injective cogenerator ${ }_{R} E$. Then $S$ Hom $R(S, E)$ is an injective cogenerator which is finitely cogenerated by [12, Proposition 5.3]. Hence $s \operatorname{Hom}_{R}(S, E)$ is artinian, since $s U$ is an artinian cogenerator. By [12, Theorem 1.2], ${ }_{R} \operatorname{Hom}_{R}(S, E)$ is also artinian. It follows from the above lemma that ${ }_{R} E$ is artinian. Hence ${ }_{R} E$ defines a duality. 
Corollary 20. Let $R \leqslant S$ be a finite triangular extension. If $S$ is a left artinian ring with a duality, then $R$ is a left artinian ring with a duality.

Proof: Using [21, Theorem 5.2] we see $R$ is a left artinian ring. Hence $R$ has a duality by Proposition 19 .

\section{REFERENCES}

[1] F.W. Anderson and K.R. Fuller, Rings and categories of modules, 2nd edition (SpringerVerlag, Berlin, Heidelberg and New York, 1992).

[2] G. Azumaya, 'A duality theory for injective modules', Amer. J. Math. 81 (1959), 249-278.

[3] G. Azumaya, 'Exact and serial rings', J. Algebra 85 (1983), 477-489.

[4] G. Azumaya, 'Characterizations of semi-perfect and perfect modules', Math. Z. 140 (1974), 95-103.

[5] P.M. Cohn, 'On a class of binomial extensions', Mlinois J. Math. 10 (1966), 418-424.

[6] R.C. Courter, 'Finite dimensional right duo algebras are duo', Proc. Amer. Math. Soc. 84 (1982), 157-161.

[7] J.W. Fisher, 'Nil subrings of endomorphism rings of modules', Proc. Amer. Math. Soc. 34 (1972), 75-78.

[8] J.W. Fisher, 'Finiteness conditions for projective and injective modules', Proc. Amer. Math. Soc. 40 (1973), 389-394.

[9] D.A. Hill, 'Endomorphism rings of hereditary modules', Arch. Math. 28 (1977), 45-50.

[10] D.A. Hill, 'Rings whose indecomposable injective modules are uniserial', Canad. J. Math. 34 (1982), 797-805.

[11] F. Kasch, Modules and rings (Academic Press, London and New York, 1982).

[12] B. Lemonnier, 'Dimension de Krull et dualite de Morita dans les extensions triangulaires', Comm. Algebra 12 (1984), 3071-3110.

[13] K. Morita, 'Duality for modules and its applications to the theory of rings with minimum condition', Tokyo Kyoiku Daigaku Ser A6 (1958), 83-142.

[14] B.J. Mueller, 'On Morita duality', Canad. J. Math. 21 (1969), 1338-1347.

[15] B.J. Mueller, 'Linear compactness and Morita duality', J. Algebra 16 (1970), 60-66.

[16] J.J. Rotman, An introduction to homological algebra (Academic Press, London and New York, 1979).

[17] A. Rosenberg and D. Zelinsky, 'Finiteness of the injective hull', Math. Z. 70 (1959), 372-380.

[18] M.S. Shrikhande, 'On hereditary and cohereditary modules', Canad. J. Math. 25 (1973), 892-896.

[19] A.K. Tiwary and B.M. Pandeya, 'Modules whose nonzero endomorphisms are monomorphisms', in Algebra and its applications, (H.L. Manocha and J.B. Srivastava, Editors), Lecture Notes Pure Appl. Math. 91 (Marcel Dekker, New York, 1984), pp. 199-203.

[20] P. Vamos, 'Rings with duality', Proc. London Math. Soc. 35 (1977), 275-289.

[21] E.A. Whelan, 'Finite subnormalizing extensions of rings', J. Algebra 101 (1986), 418-432. 
[22] Weimin Xue, 'Morita duality and artinian left duo rings', Bull. Austral. Math. Soc. 39 (1989), 339-342.

[23] Weimin Xue, 'A note on exact hereditary rings', Saitama Math. J. 7 (1989), 1-4.

[24] Weimin Xue, 'Morita duality and intermediate triangular extensions', Yokohama Math. J. 37 (1989), 57-59.

[25] Weimin Xue, Rings with morita duality, Lect. Notes. Math. 1523 (Springer-Verlag, Berlin, Heidelberg, New York, 1992).

Department of Mathematics

Fujian Normal University

Fuzhou

Fijian 350007

People's Republic of China 